\title{
Stable systolic category of the product of spheres
}

\author{
HOIL RYU
}

\begin{abstract}
The stable systolic category of a closed manifold $M$ indicates the complexity in the sense of volume. This is a homotopy invariant, even though it is defined by some relations between homological volumes on $M$. We show an equality of the stable systolic category and the real cup-length for the product of arbitrary finite dimensional real homology spheres. Also we prove the invariance of the stable systolic category under the rational equivalences for orientable 0 -universal manifolds.
\end{abstract}

$57 \mathrm{~N} 65 ; 53 \mathrm{C} 23,55 \mathrm{M} 30$

\section{Introduction}

In this paper, a manifold is assumed to be closed, connected, orientable and smooth. The systole of a manifold $M$ is the least length of non-contractible closed loops in $M$. One can generalize this concept to the least volume of $k$-dimensional non-zero homology classes, called the homology systole. Now we can imagine such systoles have some kind of relations with the entire volume of $M$, and it is natural to ask what kind of relationship exists.

As an answer, Gromov proved a theorem which says that the existence of non-trivial cup product implies the existence of the stable isosystolic inequality as follows.

Gromov's Theorem [7, 7.4.C] Let $M$ be an $n$-manifold. If there exist some reduced real cohomology classes $\alpha_{1}^{*}, \ldots, \alpha_{k}^{*}$ with $\alpha_{i}^{*}$ in $\widetilde{H}^{d_{i}}(M ; \mathbb{R})$ and a non-zero cup product $\alpha_{1}^{*} \smile \cdots \smile \alpha_{k}^{*}$ in $\widetilde{H}^{n}(M ; \mathbb{R})$, then there exists $C>0$ satisfying

$$
\prod_{i=1}^{k} \operatorname{stsys}_{d_{i}}(M, \mathcal{G}) \leq C \cdot \operatorname{mass}([M], \mathcal{G})
$$

for all Riemannian metrics $\mathcal{G}$ on $M$ where stsys $_{d_{i}}$ is the stable $d_{i}$-systole and $[M]$ is the fundamental class of $M$ with coefficients in $\mathbb{Z} / 2 \mathbb{Z}$.

The greatest $k$ satisfying the stable isosystolic inequality is called the stable systolic category of $M$, which was introduced by Katz and Rudyak [8], and is known to 
be a homotopy invariant by Katz and Rudyak [9]. We will show the stable systolic category of a 0 -universal manifold is also invariant under the rational equivalences in Corollary 4.3.

For an orientable manifold $M$, Gromov's Theorem implies that the stable systolic category is not smaller than the real cup-length. So, is there some manifold $M$ such that the stable systolic category is greater than the real cup-length? If such $M$ exists, then the inversion of Gromov's Theorem will fail for $M$. This interesting question is not answered yet, but equality is known for some manifolds, see Dranishnikov and Rudyak [3] for example. In this paper, we also show more equality later in Theorem 3.6 and Theorem 3.8.

\subsection{Definition of the stable systolic category}

To define the stable systolic category, we need to consider the flat homology theory as a metric space whose metric structure is induced by the integration on the space. One can see the details about currents and homological integration in Federer $[4 ; 5]$, Federer and Fleming [6], Serre [10] and White [11]. Since we use the integration theory to define the norm on real homology vector space, we consider the local Lipschitz category $\mathfrak{L}$ whose objects are pairs of local Lipschitz neighborhood retracts in some finite dimensional Euclidean space and whose morphisms are locally Lipschitzian maps. One can find the formal definition of $\mathfrak{L}$ in Federer [4, 4.1.29 and 4.4.1]. In this section, we define some notations of flat homology theory on $\mathfrak{L}$ briefly and define systoles and systolic category for a manifold.

Let $(X, A)$ be an object of $\mathfrak{L}$. Then we can assume that $X$ and $A$ possess the restricted metrics of $\mathbb{R}^{n}$. Let $G$ be a $\mathbb{Z}$-module with a norm $|\cdot|$ which makes $G$ a complete metric space. If $G$ is $\mathbb{Z}$ or $\mathbb{R}$, we assume that norm of $G$ is the standard norm. The comass of a differential form $\omega$ on $X$ is defined as

$$
\operatorname{comass}(\omega):=\sup \left\{\left|\omega_{x}(\tau)\right|: x \in X \text {, orthonormal } q \text {-frame } \tau\right\} .
$$

Also, the mass of a $q$-current $T$ in $X$ is the dual norm of comass, that is,

$$
\operatorname{mass}(T):=\sup \{T(\omega): \text { differential } q \text {-form } \omega, \operatorname{comass}(\omega) \leq 1\} .
$$

A Lipschitzian singular $q$-cube $\kappa: I^{q} \rightarrow X$, induces a homomorphism $\kappa_{\mathrm{b}}$ from the module of polyhedral chains $\mathcal{P}_{q}(X ; G)$ to the module of rectifiable currents $\mathcal{R}_{q}(X ; G)$. Then the mass of $\kappa$ is defined by the mass of the image $\kappa_{b} I^{q}$ where $I^{q}$ is the corresponding polyhedral $q$-current of the unit rectangular parallelepiped $I^{q}$. This correspondence of $\kappa$ to $\kappa_{\mathrm{b}} I^{q}$ gives a chain map $\Phi$ of degree 0 from the chain complex of all Lipschitzian singular cubes into the chain complex of flat chains $\mathcal{F}_{*}\left(\mathbb{R}^{n} \mid X ; G\right)$. 
Here $\mathcal{F}_{*}\left(\mathbb{R}^{n} \mid X ; G\right)$ denotes the submodule of the flat chains $\mathcal{F}_{*}\left(\mathbb{R}^{n} ; G\right)$ in $\mathbb{R}^{n}$ which consists of all flat chains supported in $X$. Then one can verify that $\Phi$ induces an isomorphism $\Phi_{*}$ from the singular homology module $H_{q}(X, A ; G)$ to the homology module $H_{q}^{b}(X, A ; G)$ of the flat chains which is called the flat homology.

For a Lipschitzian singular chain $c$, there exists a representation $\sum_{i} \kappa_{i} \otimes g_{i}$ where $g_{i}$ is contained in $G$ and $\kappa_{i}$ is a Lipschitzian singular $q$-cube which is not overlapping each other (subdivide if necessary). Then the mass of $c$ is defined as

$$
\operatorname{mass}(c):=\sum_{i}\left|g_{i}\right| \cdot \operatorname{mass}\left(\kappa_{i}\right) \text {. }
$$

The mass or volume of a singular homology class $\eta$ in $H_{q}(X, A ; G)$ is defined by

$$
\operatorname{mass}(\eta ; G):=\inf \{\operatorname{mass}(c): \eta=[c], c \text { is a Lipschitzian cycle }\} .
$$

If $G$ is $\mathbb{R}$, the mass is a norm on the homology vector spaces. We will omit $G$ in the case of $\mathbb{Z}$.

The $q$-dimensional homology systole of $(X, A)$ is defined by infimum of mass of non-trivial $q$ th integral homology classes. However Gromov [2, page 301] claims that Gromov's Theorem will fail for $S^{1} \times S^{3}$, if we consider the homology systoles instead of the stable systoles. Briefly, we can consider the stable systole as a systole in the real homology vector spaces. Here we give formal definition for the stable systole. The inclusion $\iota: \mathbb{Z} \rightarrow \mathbb{R}$ induces the coefficient homomorphism $\iota_{*}$ on homology. The stable mass on $H_{q}(X, A ; \mathbb{Z})$ is defined as the mass of the image $\iota_{*} \eta$. Then we can define the $q$-dimensional stable systole of $(X, A)$ as

$$
\operatorname{stsys}_{q}(X, A):=\inf \left\{\operatorname{stmass}(\eta): \eta \in H_{q}(X, A ; \mathbb{Z}), \iota_{*} \eta \neq 0\right\} .
$$

A homology $q$-systole or a stable $q$-systole is called trivial, if it is infinite. If the $q$ th real homology vector space $H_{q}(X, A ; \mathbb{R})$ is zero, then the stable $q$-systole is trivial for all Riemannian metrics on $(X, A)$. Hence if the $q$ th integral homology module $H_{q}(X, A ; \mathbb{Z})$ is a torsion module, then the stable $q$-systole is trivial for every metric on $(X, A)$.

For a given positive integer $n>0$, a $k$-tuple $P=\left(p_{1}, \ldots, p_{k}\right)$ of positive integers is called a partition of $n$ if $n=p_{1}+\cdots+p_{k}$ and $p_{1} \leq \cdots \leq p_{k} \leq n$. A partition $P$ is called positive (or non-negative) if $p_{i}>0$ (or $p_{i} \geq 0$ ) for all $i$. The size of a partition which denoted by $\operatorname{size}(P)$ is defined by the cardinality of positive integers contained in the partition. Hence if a $k$-tuple $P$ is a positive partition, then the size of partition is $k$. From now on, we suppose a partition is positive unless otherwise stated. For a partition $P$, the duplicated number of $p_{i}$ is the cardinality number of elements in $P$ who are equal to $p_{i}$. 
Now we define concepts for an $n$-manifold $M$. A partition $P$ of $n$ is called stable systolic categorical for $M$, if there exists a real number $C>0$ and non-trivial stable $p_{i}$-systoles such that

$$
\prod_{i=1}^{\operatorname{size}(P)} \operatorname{stsys}_{p_{i}}(M, \mathcal{G}) \leq C \cdot \operatorname{mass}([M], \mathcal{G} ; \mathbb{Z} / 2 \mathbb{Z})
$$

for every Riemannian metric $\mathcal{G}$ on $M$ with fundamental class $[M] \in H_{n}(M ; \mathbb{Z} / 2 \mathbb{Z})$.

Definition 1.1 The stable systolic category of $M$ is defined by

$\operatorname{cat}_{\text {stsys }}(M):=\sup (\{\operatorname{size}(P): P$ is stable systolic categorical partition for $M\} \cup\{0\})$.

As we said before, the real cup-length is a lower estimate for the stable systolic category from Gromov's Theorem, where the real cup-length of $M$ is defined by

$$
\operatorname{cup}_{\mathbb{R}}(M):=\min \left\{k \geq 0: \alpha_{0} \smile \alpha_{1} \smile \cdots \smile \alpha_{k}=0 \text { for all } \alpha_{i} \in \widetilde{H}^{*}(M ; \mathbb{R})\right\}
$$

and $\widetilde{H}^{*}(M ; \mathbb{R})$ denotes the reduced real cohomology ring of $M$.

If $M$ is non-orientable, then the top dimensional real cohomology vector space $H^{n}(M ; \mathbb{R})$ vanishes. So every cohomology class in $H^{n}(M ; \mathbb{R})$ vanishes, we can not apply Gromov's Theorem for top dimension. This is a reason to consider only orientable manifolds in this paper.

\subsection{Acknowledgments}

The author expresses gratitude to Professor Norio Iwase, whose guidance and support to write this paper.

\section{Preliminaries on the stable systoles}

Many equations and inequalities for mass are studied. One can find those results at Babenko [1], Federer [4] and Whitney [12]. Here we state or recall some of them for the stable systoles, with some appropriate modifications applied. Through this section, we suppose $U$ and $V$ be open subsets of $\mathbb{R}^{m}$ and $\mathbb{R}^{n}$ respectively.

Proposition 2.1 For a non-empty local Lipschitz neighborhood retract $X$ in $\mathbb{R}^{n}$, the stable 0 -systole is 1 . 
Proof Let $\mathcal{D}_{0}(X)$ be the vector space of 0-currents. A map $\mathfrak{d}: X \rightarrow \mathcal{D}_{0}(X)$ can be defined as $\mathfrak{d}(x)(\omega)=\mathfrak{d}_{x}(\omega):=\omega(x)$ for a point $x$ of $X$ and a differential 0 -form $\omega$ on $X$. Then $\mathfrak{d}_{x}$ is a polyhedral 0 -current with mass $\left(\mathfrak{d}_{x}\right)=1$. This implies that $\mathfrak{d}_{x}$ is a normal 0 -cycle with coefficients $\mathbb{Z}$. Furthermore, the image $\iota_{*} \Phi_{*}^{-1}\left[\mathfrak{d}_{x}\right]$ is not vanished in $H_{0}(X ; \mathbb{R})$. So we have

$$
\operatorname{stsys}_{0}(X)=\operatorname{mass}\left(\iota_{*} \Phi_{*}^{-1}\left[\mathfrak{d}_{x}\right]\right)=1
$$

for an arbitrary point $x$ in $X$.

Lemma 2.2 For a local Lipschitz neighborhood retract $X$ in $\mathbb{R}^{n}$, if one rescale the standard metric $\mathcal{G}$ on $\mathbb{R}^{n}$ by the square of a real number $t>0$, then the quotient mass of a homology class $\eta \in H_{q}(X ; G)$ increase by the $t^{q}$ times. Furthermore, the stable $q$-systole satisfies

$$
\operatorname{stsys}_{q}\left(X, t^{2} \mathcal{G} \mid X\right)=t^{q} \cdot \operatorname{stsys}_{q}(X, \mathcal{G} \mid X)
$$

where $\mathcal{G} \mid X$ is the restriction of $\mathcal{G}$ on $X$.

Proof A similar result was introduced by Whitney [12] for the real flat chains. So the first result is satisfied for an arbitrary homology class. Also the definition of the stable systole implies

$$
\operatorname{stsys}_{q}\left(X, t^{2} \mathcal{G} \mid X\right)=\inf \left\{t^{q} \cdot \operatorname{mass}\left(\iota_{*} \eta, \mathcal{G} \mid X ; \mathbb{R}\right): \eta \in H_{q}(X, A ; \mathbb{Z}), \iota_{*} \eta \neq 0\right\}
$$

which means the equality for the stable systoles.

Proposition 2.3 (Whitney [12, X.6 and X.7]) For a locally Lipschitzian map $f: U \rightarrow V$ and an integral rectifiable $q$-current $T$ whose support is contained in a compact subset $K$ of $U$, there exists an inequality

$$
\operatorname{mass}\left(f_{\mathrm{b}} T\right) \leq \operatorname{Lip}(f \mid K)^{q} \cdot \operatorname{mass}(T)
$$

where $\operatorname{Lip}(f \mid K)$ is the lower bound of Lipschitz constants of the restriction $f \mid K$.

Proposition 2.4 If $f:(X, A) \rightarrow(Y, B)$ is a locally Lipschitzian map, then for any homology class $\eta$ of $H_{q}(X, A ; G)$, there is a compact subset $K$ of $\mathbb{R}^{m}$ which satisfies

$$
0 \leq \operatorname{mass}\left(f_{*} \eta ; G\right) \leq \operatorname{Lip}(f \mid K)^{q} \cdot \operatorname{mass}(\eta ; G)
$$

where $f_{*}: H_{q}(X, A ; G) \rightarrow H_{q}(Y, B ; G)$ is the induced homomorphism. 
Proof Note that $f$ induces a homomorphism $f_{b}: Z_{q}(X, A ; G) \rightarrow Z_{q}(Y, B ; G)$ on flat cycles as well as $f_{\mathrm{b}} \mathcal{F}_{q}\left(\mathbb{R}^{m} \mid A ; G\right) \subset \mathcal{F}_{q}\left(\mathbb{R}^{n} \mid B ; G\right)$. For a given flat homology class $\Phi_{*} \eta$, let $T$ be a representative normal $q$-cycle in $Z_{q}(X, A ; G)$. The naturality of $\Phi_{*}$ implies $\Phi_{*} f_{*} \eta=f_{*} \Phi_{*} \eta=f_{*}[T]=\left[f_{\mathrm{b}} T\right]$. Also the relation of cosets $\left[f_{\mathrm{b}} T\right]=$ $\left[f_{\mathrm{b}} T+f_{\mathrm{b}} \mathcal{F}_{q}\left(\mathbb{R}^{m} \mid A ; G\right)\right]=\left[f_{\mathrm{b}} T+\mathcal{F}_{q}\left(\mathbb{R}^{n} \mid B ; G\right)\right]$ implies that the relation of the sets

$$
\left\{f_{\mathrm{b}} T:[T]=\Phi_{*} \eta\right\} \subset\left\{S:[S]=\Phi_{*} f_{*} \eta\right\} \subset Z_{q}(Y, B ; G) .
$$

With the definition of the mass of homology class, we obtain

$$
\operatorname{mass}\left(f_{*} \eta ; G\right) \leq \inf \left\{\operatorname{mass}\left(f_{\mathrm{b}} T\right):[T]=\Phi_{*} \eta\right\} .
$$

Because of $T$ is compact supported, there is a compact subset $K$ of $\mathbb{R}^{m}$ with $\operatorname{supp}(T) \subset$ $\operatorname{Int}(K)$. Here we can apply Proposition 2.3 for $T$, so we have

$$
\operatorname{mass}\left(f_{*} \eta ; G\right) \leq \operatorname{Lip}(f \mid K)^{q} \cdot \inf \left\{\operatorname{mass}(T):[T]=\Phi_{*} \eta\right\}
$$

which implies the result.

Lemma 2.5 Let $(X, A)$ and $(Y, B)$ are local Lipschitz neighborhood retract pairs. If a locally Lipschitzian map $f:(X, A) \rightarrow(Y, B)$ induces a monomorphism

$$
f_{*}: H_{q}(X, A ; \mathbb{R}) \rightarrow H_{q}(Y, B ; \mathbb{R}),
$$

then there is a compact subset $K$ in the ambient space of $X$ satisfying

$$
\operatorname{stsys}_{q}(Y, B) \leq \operatorname{Lip}(f \mid K)^{q} \cdot \operatorname{stsys}_{q}(X, A) .
$$

Furthermore, if $H_{q}(X, A ; \mathbb{R})$ is non-zero, then $\operatorname{stsys}_{q}(Y, B)$ is a positive real number.

Proof Proposition 2.4 and $f_{*}\left(H_{q}(X, A ; \mathbb{R}) \backslash\{0\}\right) \subset\left(H_{q}(Y, B ; \mathbb{R}) \backslash\{0\}\right)$ imply the existence of inequality in the stable systole level.

For integral homology class $\eta$ with $\iota_{*} \eta$ is non-zero, the image $f_{*} \iota_{*} \eta$ does not vanish, since $f_{*}$ is a monomorphism. Recall that the mass of real homology classes is a norm, hence $\operatorname{mass}\left(f_{*} \iota_{*} \eta\right)$ is a positive real number. Furthermore, the stable $q$-systole does not converge to zero, since $\mathbb{Z}$ is discrete.

Let $\mathcal{K}(U)$ be the set of all real valued compact supported continuous functions on $U$. We denote $\mathcal{K}^{+}(U)$ the subset of non-negative valued functions. For a subset $A$ of $U$, we say a sequence of functions $f_{1}, f_{2}, \ldots$ in $\mathcal{K}(U)$ suits $A$, if $f_{i}(x) \leq f_{i+1}(x)$ and $\lim _{i \rightarrow \infty} f_{i}(x) \geq 1$ for every $x$ in $A$. 
For a rectifiable current $T$ in $\mathcal{R}_{q}(U)$ and a function $f$ in $\mathcal{K}^{+}(U)$, a monotone Daniell integral $\|T\|$ can be defined by

$$
\|T\|(f):=\sup \left\{T(\omega): \operatorname{comass}\left(\omega_{x}\right) \leq f(x) \text { for all } x \in U\right\}
$$

where the supremum is taken over all compact supported differential $q$-forms $\omega$ on $U$. In addition, there is associated Radon measure

$$
\rho_{T}(A):=\inf \left\{\lim _{i \rightarrow \infty}\|T\|\left(f_{i}\right): f_{1}, f_{2}, \ldots \text { suits } A\right\}
$$

for a subset $A$ of $U$, which satisfying

$$
\|T\|(f)=\int_{U} f d \rho_{T} .
$$

If we consider a function $1_{U}$ which is defined by $1_{U}(x)=1$ for all $x$, the mass is obtained by $\rho_{T}$ as

$$
\rho_{T}(U)=\|T\|\left(1_{U}\right)=\operatorname{mass}(T) .
$$

One can find more details about these arguments in Federer [4, 2.5 and 4.1].

Proposition 2.6 For rectifiable currents $S$ in $\mathcal{R}_{p}(U)$ and $T$ in $\mathcal{R}_{q}(V)$, the mass of their cross product is equal to the multiplication of their masses, that is,

$$
\operatorname{mass}(S \times T)=\operatorname{mass}(S) \cdot \operatorname{mass}(T)
$$

with respect to the product metric on $U \times V$.

Proof Since $S$ and $T$ are rectifiable currents, mass can be written by associated Radon measures $\rho_{S}, \rho_{T}$ and $\rho_{S \times T}$. Therefore Fubini's Theorem (see Federer [4, 2.6.2.(2)]) implies

$$
\operatorname{mass}(S \times T)=\rho_{S \times T}(U \times V)=\rho_{S}(U) \cdot \rho_{T}(V)=\operatorname{mass}(S) \cdot \operatorname{mass}(T) .
$$

Lemma 2.7 Let $(X, A)$ and $(Y, B)$ are local Lipschitz neighborhood retract pairs. For homology classes $\xi \in H_{p}(X, A ; G)$ and $\eta \in H_{q}(Y, B ; G)$, we can estimate

$$
\begin{aligned}
\operatorname{mass}(\xi \times \eta ; G) & \leq \operatorname{mass}(\xi ; G) \cdot \operatorname{mass}(\eta ; G) \\
\operatorname{stsys}_{p+q}((X, A) \times(Y, B)) & \leq \operatorname{stsys}_{p}(X, A) \cdot \operatorname{stsys}_{q}(Y, B)
\end{aligned}
$$

and

with respect to the product metric on $(X, A) \times(Y, B)$.

Proof Let $S$ and $T$ be representative rectifiable cycles corresponding to $\xi$ and $\eta$ respectively, that is, $\Phi_{*} \xi=[S]$ with $S \in Z_{p}^{b}(X, A ; G)$ and $\Phi_{*} \eta=[T]$ with $T \in$ $Z_{q}^{b}(Y, B ; G)$. Then the naturality of a cross product implies that there is a representative 
rectifiable current with the form of a cross product $S \times T$ in the coset $[c]=\Phi_{*}(\xi \times \eta)$. Therefore

$$
\begin{aligned}
\left\{S \times T:[S] \times[T]=\Phi_{*} \xi \times \Phi_{*} \eta\right\} & =\left\{S \times T:[S \times T]=\Phi_{*}(\xi \times \eta)\right\} \\
& \subset\left\{c:[c]=\Phi_{*}(\xi \times \eta)\right\} \\
& \subset Z_{p+q}^{b}((X, A) \times(Y, B) ; G) .
\end{aligned}
$$

Hence Proposition 2.6 implies an inequality

$$
\begin{aligned}
\operatorname{mass}(\xi \times \eta ; G) & \left.\leq \inf \left\{\operatorname{mass}(S \times T):[S] \times[T]=\Phi_{*} \xi \times \Phi_{*} \eta\right)\right\} \\
& =\operatorname{mass}(\xi ; G) \cdot \operatorname{mass}(\eta ; G)
\end{aligned}
$$

on homology level. To show the inequality of the stable systoles, recall that the cross product homomorphism

$$
H_{p}(X, A ; \mathbb{R}) \otimes H_{q}(Y, B ; \mathbb{R}) \rightarrow H_{p+q}((X, A) \times(Y, B) ; \mathbb{R})
$$

is a monomorphism. Therefore we can estimate the stable $q$-systole as

$$
\begin{aligned}
\operatorname{stsys}_{p+q}((X, A) \times(Y, B)) & \leq \inf \left\{\operatorname{mass}(\xi \times \eta): \begin{array}{l}
\xi \in H_{p}(X, A ; \mathbb{Z}), \iota_{*} \xi \neq 0, \\
\eta \in H_{q}(Y, B ; \mathbb{Z}), \iota_{*} \eta \neq 0
\end{array}\right\} \\
& \leq \operatorname{stsys}_{p}(X, A) \cdot \operatorname{stsys}_{q}(Y, B) .
\end{aligned}
$$

where the second inequality is obtained by the result on homology level.

Lemma 2.8 Suppose $X$ and $Y$ are local Lipschitz neighborhood retracts. If $Y$ is connected and the Künneth formula gives an isomorphism of non-trivial vector spaces

$$
H_{q}(X ; \mathbb{R}) \otimes H_{0}(Y ; \mathbb{R}) \cong H_{q}(X \times Y ; \mathbb{R}) \neq\{0\},
$$

then the stable $q$-systole satisfies

$$
0<\operatorname{stsys}_{q}(X \times Y)=\operatorname{stsys}_{q}(X)<\infty .
$$

with respect to the product metric on $X \times Y$.

Proof Let $\mathfrak{p r}_{1}: X \times Y \rightarrow X$ be the first projection. From the assumption, for a non-zero homology class $\eta$ in $H_{q}(X \times Y ; \mathbb{R})$, there exist $[S] \neq 0$ in $H_{q}^{\mathrm{b}}(X ; \mathbb{R})$ and $[T] \neq 0$ in $H_{0}^{b}(Y ; \mathbb{R})$ whose cross product is the image of $\eta$ in $H_{q}^{b}(X \times Y ; \mathbb{R})$ with the same positive mass, that is,

$$
\operatorname{mass}([S] \times[T])=\operatorname{mass}(\eta)>0 .
$$

Note that the vector space of normal 0 -chains $\mathcal{N}_{0}(Y ; \mathbb{R})$ is equal to the vector space of polyhedral 0 -chains $\mathcal{P}_{0}(Y ; \mathbb{R})$ which is generated by $\left\{\mathfrak{d}_{y}: y \in Y\right\}$ where $\mathfrak{d}$ is defined in 
the proof of Proposition 2.1. For all points $y$ and $y^{\prime}$ in $Y,\left[\mathfrak{d}_{y}\right]=\left[\mathfrak{d}_{y^{\prime}}\right]$ implies that there is a non-zero real number $r$ such that $[T]=r\left[\mathfrak{d}_{y}\right]$ with mass $[T]=|r| \cdot \mathfrak{d}_{y}\left(1_{Y}^{*}\right)=|r|$. Also, every $[S] \times[T]$ has representation of $[r \cdot S] \times\left[\mathfrak{d}_{y}\right]$, therefore $\mathfrak{p r}_{1 *}$ is an isomorphism with $\mathfrak{p r}_{1 *}([S] \times[T])=[r \cdot S]$. Hence Lemma 2.5 implies

$$
\operatorname{stsys}_{q}(X \times Y) \geq \operatorname{stsys}_{q}(X)>0
$$

with the fact of $\mathfrak{p r}_{1}$ is a Lipschitzian map with $\operatorname{Lip}\left(\mathfrak{p r}_{1}\right)=1$. As a result, we obtain the equality by combining the result of Lemma 2.7 .

\section{Calculation by dimension and constructing metrics}

At first, we will calculate the stable systolic category from the dimensional information of homology. If the homology group is not so complex, such as in the case of a real homology sphere, we know the stable systolic category by only using dimensional information. If an oriented manifold has a relatively simple cup-product structure such as $n$-fold producted space of spheres, then the stable systolic category can be also calculated instantly. Such methods to calculate the stable systolic category can be generalized as follows.

For a topological space $X$, let $\operatorname{lpd}(X)$ denote the least positive dimension of real cohomology vector spaces of $X$. So $\operatorname{lpd}(X)=l$ if and only if $\widetilde{H}^{i}(X ; \mathbb{R})=\{0\}$ for $0<i<l$ and $\widetilde{H}^{l}(X ; \mathbb{R}) \neq\{0\}$. If $M$ is an $m$-manifold, then $\operatorname{lpd}(M)$ is less than or equal to $m$.

Definition 3.1 An $n$-dimensional $\mathrm{CW}$ space $X$ is said to have maximal real cup length, if there exist some real cohomology classes $\alpha_{1}, \ldots, \alpha_{r}$ with $\alpha_{i} \in \widetilde{H}^{d_{i}}(X ; \mathbb{R})$, a non-zero cup-product $\alpha_{1} \smile \cdots \smile \alpha_{r} \in \widetilde{H}^{n}(X ; \mathbb{R})$ and $r:=\lfloor n / \operatorname{lpd}(X)\rfloor$ where $\lfloor x\rfloor$ denotes the floor of a real number $x$.

Example Let $S$ be a manifold which is a real homology sphere. Then $S$ has maximal real cup length, because of $\operatorname{lpd}(S)=\operatorname{dim}(S)$. The $n$-fold direct product of $S$ also has maximal real cup length. The direct product $S^{2} \times S^{3}$ of spheres has maximal real cup length.

Corollary 3.2 If an $m$-manifold $M$ has maximal real cup length, then the stable systolic category of $M$ is equal to the real cup-length of $M$, that is,

$$
\operatorname{cat}_{\text {stsys }}(M)=\operatorname{cup}_{\mathbb{R}}(M)=\lfloor m / \operatorname{lpd}(M)\rfloor .
$$


Proof We need to verify that cat $\operatorname{stsys}(M) \leq \operatorname{cup}_{\mathbb{R}}(M)$. Let $r:=\lfloor m / \operatorname{lpd}(M)\rfloor$. If $\left(d_{1}, \ldots, d_{k}\right)$ is a partition of $m$ such that each stable $d_{i}$-systole is non-trivial, then $d_{i} \geq \operatorname{lpd}(M)$, so there is an inequality

$$
k \cdot \operatorname{lpd}(M) \leq m=d_{1}+\cdots+d_{k}<(r+1) \cdot \operatorname{lpd}(M)
$$

which implies $k \leq r=\operatorname{cup}_{\mathbb{R}}(M)$.

In general, the direct product $M \times N$ of manifolds does not have maximal real cup length even if $M$ and $N$ have maximal real cup-length. For example, the direct product of spheres $S^{1} \times S^{2}$ does not have maximal real cup length.

Lemma 3.3 If manifolds $M_{1}^{m_{1}}, \ldots, M_{n}^{m_{n}}$ have maximal real cup length, then the stable systolic category of their $n$-fold direct product $M_{1} \times \cdots \times M_{n}$ is greater than the sum of stable systolic categories for each $M_{i}$, that is,

$$
\text { cat }_{\text {stsys }}\left(M_{1} \times \cdots \times M_{n}\right) \geq \text { cat }_{\text {stsys }}\left(M_{1}\right)+\cdots+\text { cat }_{\text {stsys }}\left(M_{n}\right) .
$$

Proof Since $M_{i}$ has maximal real cup length, there is non-zero cup product $\alpha_{i, 1} \smile$ $\cdots \smile \alpha_{i, r_{i}}$ in $H^{m_{i}}\left(M_{i} ; \mathbb{R}\right)$ where $r_{i}:=\left\lfloor m_{i} / \operatorname{lpd}\left(M_{i}\right)\right\rfloor=$ cat $_{\text {stsys }}\left(M_{i}\right)$ for $1 \leq i \leq n$.

By the Künneth formula, the $n$-fold cross product on the top dimensions induces an isomorphism

$$
\bigotimes_{i=1}^{n} H^{m_{i}}\left(M_{i} ; \mathbb{R}\right) \cong H^{m}\left(M_{1} \times \cdots \times M_{n} ; \mathbb{R}\right) \quad \text { where } m:=\sum_{i=1}^{n} m_{i}
$$

This implies that the cross product of all $\alpha_{i, 1} \smile \cdots \smile \alpha_{i, r_{i}}$ is non-zero which can be written as a cup product

$$
\smile_{i=1}^{n} \mathfrak{p r}_{i}^{*}\left(\alpha_{i, 1} \smile \cdots \smile \alpha_{i, r_{i}}\right)=\mathfrak{p r}_{1}^{*} \alpha_{1,1} \smile \cdots \smile \mathfrak{p r}_{i}^{*} \alpha_{i, j_{i}} \smile \cdots \smile \mathfrak{p r}_{n}^{*} \alpha_{n, r_{n}}
$$

in the top-dimensional real cohomology vector space $H^{m}\left(M_{1} \times \cdots \times M_{n} ; \mathbb{R}\right)$, where $\mathfrak{p r}_{i}: M_{1} \times \cdots \times M_{n} \rightarrow M_{i}$ is the $i$ th projection, $1 \leq i \leq n$ and $1 \leq j_{i} \leq r_{i}$. This cup product implies that $r_{1}+\cdots+r_{n}$ is a lower estimate for the stable systolic category of $M_{1} \times \cdots \times M_{n}$ from Gromov's Theorem.

Proposition 3.4 For manifolds $M$ and $N$, the least positive dimension of cohomology of $M \times N$ is the minimum of $\operatorname{lpd}(M)$ and $\operatorname{lpd}(N)$.

Proof From the Künneth formula, the cohomology $H^{i}(M \times N ; \mathbb{R})=\{0\}$ for $0<i<$ $\min (\operatorname{lpd}(M), \operatorname{lpd}(N))$. If $l:=\min (\operatorname{lpd}(M), \operatorname{lpd}(N))=\operatorname{lpd}(M)$, then $H^{l}(M ; \mathbb{R})$ is non-zero and the cross product homomorphism

$$
H^{l}(M ; \mathbb{R}) \otimes H^{0}(N ; \mathbb{R}) \rightarrow H^{l}(M \times N ; \mathbb{R})
$$


is a monomorphism. Therefore $H^{l}(M \times N ; \mathbb{R})$ is non-zero. The case of $\operatorname{lpd}(M)>$ $\operatorname{lpd}(N)$ is shown by using the same arguments.

For integers $i$ and $j \neq 0$, let $\bmod (i, j)$ denotes the remainder from the division of $i$ by $j$.

Corollary 3.5 Suppose manifolds $M^{m}$ and $N^{n}$ have maximal real cup length, and an integer $l:=\operatorname{lpd}(M \times N)$. If $M$ and $N$ satisfy the conditions

and

$$
\lfloor m / \operatorname{lpd}(M)\rfloor=\lfloor m / l\rfloor, \quad\lfloor n / \operatorname{lpd}(N)\rfloor=\lfloor n / l\rfloor
$$

$$
\bmod (m, l)+\bmod (n, l)<l,
$$

then $M \times N$ has maximal real cup length. Therefore,

$$
\text { cat }_{\text {stsys }}(M \times N)=\text { cat }_{\text {stsys }}(M)+\text { cat }_{\text {stsys }}(N) .
$$

Proof Let integers $r:=\lfloor m / l\rfloor$ and $s:=\lfloor n / l\rfloor$.

Proposition 3.4 implies that $l=\min (\operatorname{lpd}(M), \operatorname{lpd}(N))=\operatorname{lpd}(M \times N)$. So we can formulate $\lfloor(m+n) / \operatorname{lpd}(M \times N)\rfloor=r+s+\lfloor\bmod (m, l)+\bmod (n, l)\rfloor$. By the assumption, $\lfloor\bmod (m, \operatorname{lpd}(M))+\bmod (n, \operatorname{lpd}(N))\rfloor$ is zero, so we have

$$
\lfloor(m+n) / \operatorname{lpd}(M \times N)\rfloor=r+s .
$$

Thus it is sufficient to show that there is a non-zero cup product with the length of $r+s$.

Since $M$ and $N$ have maximal real cup length, there are cohomology classes $\alpha_{1}, \ldots, \alpha_{r}$ and $\beta_{1}, \ldots, \beta_{s}$ with their cup products are non-zero cohomology classes $\alpha_{1} \smile \cdots \smile \alpha_{r}$ in $H^{m}(M ; \mathbb{R})$ and $\beta_{1} \smile \cdots \smile \beta_{s}$ in $H^{n}(M ; \mathbb{R})$. From the proof of Lemma 3.3, there is a non-zero cup product $\mathfrak{p r}_{1}^{*} \alpha_{1} \smile \ldots \smile \mathfrak{p r}_{1}^{*} \alpha_{r} \smile \mathfrak{p r}_{2}^{*} \beta_{1} \smile \ldots \smile \mathfrak{p r}_{2}^{*} \beta_{s}$ in the top dimensional cohomology vector space $H^{m+n}(M \times N ; \mathbb{R})$.

Without the condition of the product $M \times N$ has maximal real cup length, we can generalize this corollary as follow.

Theorem 3.6 Let manifolds $M^{m}$ and $N^{n}$ have maximal real cup length. If

$$
\bmod (m, \operatorname{lpd}(M))+\bmod (n, \operatorname{lpd}(N))<\max (\operatorname{lpd}(M), \operatorname{lpd}(N)),
$$

then the stable systolic category of their product $M \times N$ is the sum of each stable systolic category, that is,

$$
\mathrm{cat}_{\text {stsys }}(M \times N)=\mathrm{cat}_{\text {stsys }}(M)+\mathrm{cat}_{\text {stsys }}(N) .
$$


Proof Since $M$ and $N$ have maximal real cup length,

$$
r:=\lfloor m / \operatorname{lpd}(M)\rfloor=\operatorname{cat}_{\text {stsys }}(M) \quad \text { and } \quad s:=\lfloor n / \operatorname{lpd}(N)\rfloor=\operatorname{cat}_{\text {stsys }}(N) .
$$

In the case of $\operatorname{lpd}(M)=\operatorname{lpd}(N)$ is Corollary 3.5. So we will assume $\operatorname{lpd}(M)<\operatorname{lpd}(N)$.

From Lemma 3.3, cat stsys $(M \times N) \geq$ cat $_{\text {stsys }}(M)+$ cat $_{\text {stsys }}(N)=r+s$. Therefore, it is sufficient to show that any partition of $m+n$ whose size is greater than $r+s$, is not a stable systolic categorical partition.

Suppose the partition $\left(d_{1}, \ldots, d_{k}\right)$ of $m+n$ is a stable systolic categorical for $M \times N$ with some integer $1 \leq r^{\prime} \leq k$ and the condition $0<\operatorname{lpd}(M) \leq d_{1} \leq \cdots \leq d_{r^{\prime}}<\operatorname{lpd}(N)$. For an arbitrary $t \geq 1$, let $\mathcal{G}_{t}:=t^{2} \mathcal{G}_{M}+\mathcal{G}_{N}$ be a Riemannian metric on $M \times N$. Then Lemma 2.2 and Lemma 2.8 imply that the stable systoles for the partition $\left(d_{1}, \ldots, d_{k}\right)$ satisfies

$$
\begin{aligned}
\prod_{i=1}^{k} \operatorname{stsys}_{d_{i}}\left(M \times N, \mathcal{G}_{t}\right) & \geq \prod_{i=1}^{r^{\prime}} \operatorname{stsys}_{d_{i}}\left(M, t^{2} \mathcal{G}_{M}\right) \cdot \prod_{j=r^{\prime}+1}^{k} \operatorname{stsys}_{d_{j}}\left(M \times N, \mathcal{G}_{t}\right) \\
& =t^{d_{1}+\cdots+d_{r^{\prime}}} \cdot \prod_{i=1}^{r^{\prime}} \operatorname{stsys}_{d_{i}}\left(M, \mathcal{G}_{M}\right) \cdot \prod_{j=r^{\prime}+1}^{k} \operatorname{stsys}_{d_{j}}\left(M \times N, \mathcal{G}_{t}\right)
\end{aligned}
$$

Since $t \geq 1$, we can obtain the inequality $\operatorname{stsys}_{d_{j}}\left(M \times N, \mathcal{G}_{t}\right) \geq \operatorname{stsys}_{d_{j}}\left(M \times N, \mathcal{G}_{1}\right)$ for each $r^{\prime}+1 \leq j \leq k$. On the other hands, the mass of integral fundamental class $[M \times N]$ is characterized by Lemma 2.2 and Lemma 2.7 as

$$
\begin{aligned}
\operatorname{mass}\left([M \times N], \mathcal{G}_{t}\right) & \leq \operatorname{mass}\left([M], t^{2} \mathcal{G}_{M}\right) \cdot \operatorname{mass}\left([N], \mathcal{G}_{N}\right) \\
& =t^{m} \cdot \operatorname{mass}\left([M], \mathcal{G}_{M}\right) \cdot \operatorname{mass}\left([N], \mathcal{G}_{N}\right) .
\end{aligned}
$$

Here if we assume that $d_{1}+\cdots+d_{r^{\prime}}>m$, then we have

$$
\frac{\prod_{i=1}^{k} \operatorname{stsys}_{d_{i}}\left(M \times N, \mathcal{G}_{t}\right)}{\operatorname{mass}\left([M \times N], \mathcal{G}_{t}\right)} \geq t^{\left(d_{1}+\cdots+d_{r^{\prime}}\right)-m} \cdot \frac{\prod_{i=1}^{r^{\prime}} \operatorname{stsys}_{d_{i}}\left(M, \mathcal{G}_{M}\right) \cdot \prod_{j=r^{\prime}+1}^{k} \operatorname{stsys}_{d_{j}}\left(M \times N, \mathcal{G}_{1}\right)}{\operatorname{mass}\left([M], \mathcal{G}_{M}\right) \cdot \operatorname{mass}\left([N], \mathcal{G}_{N}\right)}
$$

where the right-hand side of the inequality diverges as $t \rightarrow \infty$. This contradicts to that $\left(d_{1}, \ldots, d_{k}\right)$ is a stable systolic categorical partition. Hence we obtain $d_{1}+\cdots+d_{r^{\prime}} \leq m$ and $d_{r^{\prime}+1}+\cdots+d_{k} \geq n$. This condition for $m$ implies

$$
r^{\prime} \leq\left\lfloor\left(d_{1}+\cdots+d_{r^{\prime}}\right) / \operatorname{lpd}(M)\right\rfloor \leq\lfloor m / \operatorname{lpd}(M)\rfloor \leq r .
$$

Let $s^{\prime}:=k-r^{\prime}$. From the assumption, $\operatorname{lpd}(M) / \operatorname{lpd}(N)<1$ and

$$
\bmod (m, \operatorname{lpd}(M))+\bmod (n, \operatorname{lpd}(N))<\operatorname{lpd}(N),
$$


so we can calculate as

$$
k=r^{\prime}+s^{\prime} \leq r+s
$$

which implies cat stsys $(M \times N) \leq$ cat $_{\text {stsys }}(M)+$ cat $_{\text {stsys }}(N)$.

Corollary 3.7 Suppose manifolds $M_{0} \times M_{1} \times \cdots \times M_{k}$ and $M_{k+1} \times \cdots \times M_{n} \times M_{n+1}$ have maximal real cup length with

$$
\operatorname{lpd}\left(M_{0}\right)=\operatorname{lpd}\left(M_{1}\right)=\cdots=\operatorname{lpd}\left(M_{k}\right)
$$

and

$$
\operatorname{lpd}\left(M_{k+1}\right)=\cdots=\operatorname{lpd}\left(M_{n}\right)=\operatorname{lpd}\left(M_{n+1}\right) .
$$

Let $r_{i}:=\left\lfloor\operatorname{dim}\left(M_{i}\right) / \operatorname{lpd}\left(M_{i}\right)\right\rfloor$ for $0 \leq i \leq n+1$. If $M_{0}, \ldots, M_{n+1}$ satisfy conditions $\operatorname{dim}\left(M_{i}\right)=\operatorname{lpd}\left(M_{i}\right) \cdot r_{i}$ for $1 \leq i \leq n$ and

$$
\begin{aligned}
\operatorname{dim}\left(M_{0}\right)-\operatorname{lpd}\left(M_{0}\right) \cdot r_{0}+\operatorname{dim}\left(M_{n+1}\right)-\operatorname{lpd}\left(M_{n+1}\right) & \cdot r_{n+1} \\
& <\max \left(\operatorname{lpd}\left(M_{0}\right), \operatorname{lpd}\left(M_{n+1}\right)\right),
\end{aligned}
$$

then

$$
\text { cat }_{\text {stsys }}\left(\prod_{i=0}^{n+1} M_{i}\right)=\sum_{i=0}^{n+1} \mathrm{cat}_{\mathrm{stsys}}\left(M_{i}\right)=\sum_{i=0}^{n+1} r_{i}
$$

Note that Theorem 3.6 is not applied for the product $S^{1} \times S^{2}$ of spheres, but we will show the equality for such partial cases as follow.

Theorem 3.8 If manifolds $S_{1}^{m_{1}}, \ldots, S_{n}^{m_{n}}$ are real homology spheres, then the stable systolic category of their $n$-fold direct product is the number of spheres.

Proof Since every real homology spheres have maximal real cup length, Lemma 3.3 gives us a lower estimate cat stsys $\left(S_{1} \times \cdots \times S_{n}\right) \geq n$.

Suppose $m_{i} \leq m_{i+1}$ for each $1 \leq i \leq n$. Then a partition $\left(m_{1}, \ldots, m_{n}\right)$ of $\sum_{i} m_{i}$ can be rewritten as $\left(r_{1}, \ldots, r_{1}, r_{2}, \ldots, r_{l-1}, r_{l}, \ldots, r_{l}\right)$ where $r_{i}$ is a range. This corresponding to rewrite

$$
\begin{aligned}
S_{1}^{m_{1}} \times \cdots \times S_{n}^{m_{n}}=\left(S_{1}^{r_{1}} \times \cdots \times S_{s_{1}}^{r_{1}}\right) \times( & \left.S_{s_{1}+1}^{r_{2}} \times \cdots \times S_{s_{1}+s_{2}}^{r_{2}}\right) \times \cdots \\
& \times\left(S_{s_{1}+\cdots+s_{l-1}+1}^{r_{l}} \times \cdots \times S_{s_{1}+\cdots+s_{l-1}+s_{l}}^{r_{l}}\right)
\end{aligned}
$$

where $r_{i}:=m_{s_{1}+\cdots+s_{i-1}+1}=\cdots=m_{s_{1}+\cdots+s_{i-1}+s_{i}}$ with $r_{i}<r_{i+1}$ and $s_{i}>0$ is the duplicated number of $r_{i}$, so that $s_{1}+\cdots+s_{l}=n$. For simplicity, let define

$$
X_{p}:=S_{1} \times \cdots \times S_{s_{1}+\cdots+s_{p}} \quad \text { and } \quad Y_{p}:=S_{s_{1}+\cdots+s_{p}+1} \times \cdots \times S_{n}
$$


for $1 \leq p \leq n$. Then $S_{1} \times \cdots \times S_{n}=X_{p} \times Y_{p}$ and we can observe that $\mathcal{G}_{p, t}:=t^{2} \mathcal{G}_{X_{p}}+\mathcal{G}_{Y_{p}}$ is a Riemannian metric on $X_{p} \times Y_{p}$ for $t>0$ when $\mathcal{G}_{X_{p}}+\mathcal{G}_{Y_{p}}$ is a Riemannian metric on $X_{p} \times Y_{p}$. Now we can apply Lemma 2.8 and Lemma 2.2, so there exist equations

$$
\operatorname{stsys}_{q}\left(X_{p} \times Y_{p}, \mathcal{G}_{p, t}\right)=\operatorname{stsys}_{q}\left(X_{p}, t^{2} \mathcal{G}_{X_{p}}\right)=t^{q} \cdot \operatorname{stsys}_{q}\left(X_{p}, \mathcal{G}_{X_{p}}\right)
$$

for the non-trivial stable systoles in the dimension of $1 \leq q \leq s_{1}+\cdots+s_{p}$.

Let $\left(d_{1}, \ldots, d_{k}\right)$ be the longest stable systolic categorical partition for $S_{1} \times \cdots \times S_{n}$ with the condition $d_{i} \leq d_{i+1}$. Then we can rewrite $\left(d_{1}, \ldots, d_{k}\right)$ by the ranges $\left\{r_{1}, \ldots, r_{l}\right\}$ with the duplicated number $s_{i}^{\prime} \geq 0$ of $r_{i}$. We will show that the partition is not longer than $n$ by induction on $p$ for $1 \leq p \leq l$ and contradiction. Assume that $s_{i}^{\prime}=s_{i}$ for $1 \leq i \leq p-1$. If $s_{p}^{\prime}>s_{p}$, then using a similar argument in the proof of Theorem 3.6, we can observe that the right-hand side of the inequality

$$
\frac{\prod_{i=1}^{k} \operatorname{stsys}_{d_{i}}\left(X_{p} \times Y_{p}, \mathcal{G}_{p, t}\right)}{\operatorname{mass}\left(\left[X_{p} \times Y_{p}\right], \mathcal{G}_{p, t}\right)} \geq t^{w} \cdot \frac{\prod_{i=1}^{p} \operatorname{stsys}_{r_{i}}\left(X_{p}, \mathcal{G}_{X_{p}}\right)^{s_{i}^{\prime}} \cdot \prod_{i=p+1}^{l} \operatorname{stsys}_{r_{i}}\left(X_{p} \times Y_{p}, \mathcal{G}_{p, 1}\right)^{s_{i}^{\prime}}}{\operatorname{mass}\left(\left[X_{p}\right], \mathcal{G}_{X_{p}}\right) \cdot \operatorname{mass}\left(\left[Y_{p}\right], \mathcal{G}_{Y_{p}}\right)}
$$

diverges as $t$ approaches $\infty$ where $w:=r_{1}\left(s_{1}^{\prime}-s_{1}\right)+\cdots+r_{p}\left(s_{p}^{\prime}-s_{p}\right)=r_{p}\left(s_{p}^{\prime}-s_{p}\right)>0$. This contradicts to that the partition $\left(d_{1}, \ldots, d_{k}\right)$ is stable systolic categorical, and hence we obtain $s_{p}^{\prime} \leq s_{p}$. However we must choose $s_{p}^{\prime}=s_{p}$ to make the longest partition. As a result, the size of the longest stable systolic categorical partition can not exceed $n=s_{1}+\cdots+s_{l}$.

\section{Invariance under the rational equivalences}

Let $U$ be an open subset of some finite dimensional Euclidean space. For a compact subset $C$ of $U$ and a flat $q$-chain $T$ in $\mathcal{F}_{q}(U \mid C ; \mathbb{R})$, the flat norm is defined by

$$
|T|_{C}^{b}:=\inf \left\{\operatorname{mass}(T-\partial S)+\operatorname{mass}(S): S \in \mathcal{F}_{q+1}(U \mid C ; \mathbb{R})\right\}
$$

where $\mathcal{F}_{q}(U \mid C)$ is the module of all flat $q$-chains in $U$ whose support is contained in $C$.

Suppose $M$ and $N$ are $n$-manifolds. Let $K$ and $L$ be a triangulation of $M$ and $N$ respectively. In this section, $K$ and $L$ are subdivided if necessary, but we will use the same symbol. For a continuous map $f: M \rightarrow N$, there is a non-degenerate simplicial approximation $g: K \rightarrow L$ of $f$. For an open $n$-simplex $e$ in $L$, consider a map $h: K \stackrel{g}{\rightarrow} L \rightarrow L /(L \backslash e)$. We will call $\operatorname{deg}(h)$ the degree of $g$ at $e$ which is denoted by $\operatorname{deg}_{e}(g)$. Let

$$
D(g):=\sup \left\{\left|\operatorname{deg}_{e}(g)\right|: \text { open } n \text {-simplex } e \text { in } L\right\} .
$$


Here $D(g)$ is finite, because of we can assume that $K$ and $L$ are finite simplicial complexes.

For an arbitrary Riemannian metric $\mathcal{G}_{N}$ on $N$, consider an embedding in $\mathbb{R}^{m}$. Then a current $V_{N}(\omega):=\int_{N} \operatorname{comass}\left(\omega_{x}\right) d \mathcal{L}^{n} x$ is defined for an arbitrary compact supported differential $n$-form $\omega$ where $\mathcal{L}^{n}$ is the $n$-dimensional Lebesgue measure. We can observe that $V_{N}$ is contained in $\mathcal{F}_{n}\left(\mathbb{R}^{m} \mid N ; \mathbb{R}\right)$ and satisfying $\operatorname{mass}\left(V_{N}\right)=\operatorname{stsys}_{n}(N)$. We take a closed $m$-ball $C$ in $\mathbb{R}^{m}$ which contains $N$ and $L$ in its internal. For a sufficiently small $\varepsilon>0$, there is a piecewise linear metric $\mathcal{G}_{L}=\mathcal{G}_{L}(\varepsilon)$ on $L$ satisfying

$$
\left|V_{L}-V_{N}\right|_{C}^{b} \leq \varepsilon \quad \text { and } \quad\left|\operatorname{stsys}_{q}\left(L, \mathcal{G}_{L}\right)-\operatorname{stsys}_{q}\left(N, \mathcal{G}_{N}\right)\right| \leq \varepsilon
$$

for every non-trivial stable $q$-systoles (compare Federer [4, 4.1.22]) and the realization of $L$ with $\mathcal{G}_{L}$ is a PL section of the normal bundle over $N$ with $\mathcal{G}_{N}$ in $\mathbb{R}^{m}$. Such metric can be obtained by subdividing $K$ and $L$, and translating vertices in $L$ along the fiber of the normal bundle to do not degenerate any simplex. For $0<\varepsilon^{\prime}<\varepsilon$, a suitable metric $\mathcal{G}_{L}\left(\varepsilon^{\prime}\right)$ also can be acquired by the same way. Hence we can assume that $D(g)$ is not changed by $\varepsilon$ and $\mathcal{G}_{L}$. As $\varepsilon$ approaches to 0 , each $L, \mathcal{G}_{L}$ and $g^{*} \mathcal{G}_{L}$ converges to $N, \mathcal{G}_{N}$ and a piecewise Riemannian metric on $M$ respectively. Under this circumstance, we obtain following lemma.

Lemma 4.1 Suppose $q$ th real homology vector space of $K$ and $L$ are non-trivial. If $g: K \rightarrow L$ induces a monomorphism $g_{*}$ between the $q$ th real homology vector spaces, then

$$
\operatorname{stsys}_{q}\left(L, \mathcal{G}_{L}\right) \leq \operatorname{stsys}_{q}\left(K, g^{*} \mathcal{G}_{L}\right) \leq D(g) \cdot \operatorname{stsys}_{q}\left(L, \mathcal{G}_{L}\right)<\infty
$$

for every piecewise linear metric $\mathcal{G}_{L}$ on $L$.

Proof With the pullback PL metric $g^{*} \mathcal{G}_{L}$ on $K, g$ is a distance decreasing map. Combining this with Lemma 2.5,

$$
\operatorname{stsys}_{q}\left(L, \mathcal{G}_{L}\right) \leq \operatorname{Lip}(g)^{q} \cdot \operatorname{stsys}_{q}\left(K, g^{*} \mathcal{G}_{L}\right) \leq \operatorname{stsys}_{q}\left(K, g^{*} \mathcal{G}_{L}\right) .
$$

On the other hands, the inverse image of an arbitrary $q$-simplex of $L$ is $D(g)$ of $q-$ simplices as at most, since $g$ is a non-degenerate simplicial map and every $q$-simplex is contained in the boundary of some $n$-simplex for $q<n$. Also each simplex in the inverse image has same mass of the preimage, since the restriction of $g$ on each simplex is isometry. This implies that the mass of a $q$-chain $c$ of $K$ is not greater than $D(g)$ times of the mass of the image $g_{b}(c)$ which is not trivial. Therefore we can verify that

$$
\operatorname{stsys}_{q}\left(K, g^{*} \mathcal{G}_{L}\right) \leq D(g) \cdot \operatorname{stsys}_{q}\left(L, \mathcal{G}_{L}\right)
$$

for an arbitrary PL metric $\mathcal{G}_{L}$. 
Remark If $K$ is not a triangulation of a manifold, we can not sure that every $q-$ simplex of $K$ is contained in the boundary of some $n$-simplex for $q<n$. For example, a triangulation of the one-point union $S^{1} \vee S^{2}$ has some 1-simplex in $S^{1}$ which is not contained in the boundary of any 2 -simplex.

Since the stable systolic category is a homotopy invariant, here we obtain following proposition using similar techniques of Katz and Rudyak [9].

Proposition 4.2 Let $M$ and $N$ are $n$-manifolds. If there exists a smooth map $f: M \rightarrow N$ which induces a monomorphism on every real homology vector space, then cat $_{\text {stsys }}(M) \leq$ cat $_{\text {stsys }}(N)$.

Proof We apply Lemma 4.1,

$$
\operatorname{stsys}_{q}\left(N, \mathcal{G}_{N}\right) \leq \operatorname{stsys}_{q}\left(L, \mathcal{G}_{L}\right)+\varepsilon \leq \operatorname{stsys}_{q}\left(K, g^{*} \mathcal{G}_{L}\right)+\varepsilon
$$

and

$$
\operatorname{stsys}_{q}\left(N, \mathcal{G}_{N}\right)+\varepsilon \geq \operatorname{stsys}_{q}\left(L, \mathcal{G}_{L}\right) \geq 1 / D(g) \cdot \operatorname{stsys}_{q}\left(K, g^{*} \mathcal{G}_{L}\right)
$$

where $L$ converges to $N$ in some Euclidean space and $g^{*} \mathcal{G}_{L}$ converges to a piecewise Riemannian metric $\mathcal{G}_{M}$ on $M$ as $\varepsilon$ approaches to 0 . Suppose there exists a stable systolic categorical partition $\left(d_{1}, \ldots, d_{k}\right)$ for $M$. Then there exist $C>0$ and $\delta=$ $\delta(\varepsilon)>0$ such that $\delta$ converges to 0 as $\varepsilon$ approaches to 0 and

$$
\prod_{i=1}^{k} \operatorname{stsys}_{d_{i}}\left(K, g^{*} \mathcal{G}_{L}\right) \leq C \cdot \operatorname{mass}\left([K], g^{*} \mathcal{G}_{L}\right)+\delta,
$$

because of each metric $g^{*} \mathcal{G}_{L}$ can be approximated by some Riemannian metrics on $M$. We can assume that $\varepsilon \leq$ stsys $_{d_{i}}\left(N, \mathcal{G}_{N}\right)$ for all $i$, so

$$
\begin{aligned}
\prod_{i=1}^{k} \operatorname{stsys}_{d_{i}}\left(L, \mathcal{G}_{L}\right) & \leq 2^{k} \cdot \prod_{i=1}^{k} \operatorname{stsys}_{d_{i}}\left(K, g^{*} \mathcal{G}_{L}\right) \\
& \leq 2^{k} \cdot C \cdot \operatorname{mass}\left([K], g^{*} \mathcal{G}_{L}\right)+2^{k} \delta \\
& \leq 2^{k} \cdot C \cdot D(g) \cdot \operatorname{mass}\left([L], \mathcal{G}_{L}\right)+2^{k}(C \cdot D(g) \cdot \varepsilon+\delta) .
\end{aligned}
$$

This implies the partition $\left(d_{1}, \ldots, d_{k}\right)$ is also stable systolic categorical for $N$. Therefore we obtain the result cat stsys $(M) \leq$ cat $_{\text {stsys }}(N)$.

Let $X$ and $Y$ are simply connected spaces. A continuous map $f: X \rightarrow Y$ is called a rational equivalence, if the induced map $f^{*}: H^{*}(Y ; \mathbb{Q}) \rightarrow H^{*}(X ; \mathbb{Q})$ is an isomorphism. 
Corollary 4.3 The stable systolic category of a 0 -universal manifold is invariant under the rational equivalences.

Proof For a 0 -universal manifold $M$ and a rational equivalence to a space $X$, there exists a rational equivalence from $X$ to $M$.

\section{References}

[1] I K Babenko, Asymptotic invariants of smooth manifolds, Izv. Ross. Akad. Nauk Ser. Mat. 56 (1992) 707-751 MR1208148

[2] M Berger, Systoles et applications selon Gromov, Astérisque 216 (1993) 279-310 MR1246401 Séminaire Bourbaki 1992/1993, Exposé 771

[3] A N Dranishnikov, Y B Rudyak, Stable systolic category of manifolds and the cuplength, J. Fixed Point Theory Appl. 6 (2009) 165-177 MR2558490

[4] H Federer, Geometric measure theory, Die Grundlehren der mathematischen Wissenschaften 153, Springer New York, New York (1969) MR0257325

[5] H Federer, Real flat chains, cochains and variational problems, Indiana Univ. Math. J. 24 (1974/75) 351-407 MR0348598

[6] H Federer, W H Fleming, Normal and integral currents, Ann. of Math. (2) 72 (1960) 458-520 MR0123260

[7] M Gromov, Filling Riemannian manifolds, J. Differential Geom. 18 (1983) 1-147 MR697984

[8] M G Katz, Y B Rudyak, Lusternik-Schnirelmann category and systolic category of low-dimensional manifolds, Comm. Pure Appl. Math. 59 (2006) 1433-1456 MR2248895

[9] M G Katz, Y B Rudyak, Bounding volume by systoles of 3-manifolds, J. Lond. Math. Soc. (2) 78 (2008) 407-417 MR2439632

[10] J-P Serre, Homologie singulière des espaces fibrés. Applications, Ann. of Math. (2) 54 (1951) 425-505 MR0045386

[11] B White, Rectifiability of flat chains, Ann. of Math. (2) 150 (1999) 165-184 MR1715323

[12] H Whitney, Geometric integration theory, Princeton University Press, Princeton, NJ (1957) MR0087148

Graduate School of Mathematics, Kyushu University

774, Motooka, Nishi-ku, Fukuoka, 819-0395, Japan

h-ryu@math.kyushu-u.ac.jp

Received: 17 July 2010 Revised: 27 October 2010 